\title{
MINLP Model for Synthesis of Paraxylene Separation Processes Based on Crystallization Technology
}

\author{
Carlos A. Mendez ${ }^{\mathrm{a}}$, John Myers ${ }^{\mathrm{b}}$, Scott Roberts ${ }^{\mathrm{b}}$, Jeffery Logsdon $^{\mathrm{b}}$, Anastasia Vaia ${ }^{\mathrm{b}}$, \\ and Ignacio E. Grossmann ${ }^{\mathrm{a}^{*}}$ \\ ${ }^{a}$ Department of Chemical Engineering, Carnegie Mellon University, Pittsburgh,

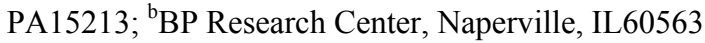

\begin{abstract}
In this paper we address the development of an MINLP optimization model for the synthesis of paraxylene separation processes based on crystallization technology. A superstructure is proposed that considers different alternative designs and operating conditions for the recovery of a high purity paraxylene product from a feed stream containing other xylene isomers and ethylbenzene. The goal is to maximize the overall annual profitability of the process, including capital and operating cost as well as product revenues. Due to the large size of the MINLP model, as well as the great difficulty in converging the corresponding equations, a solution strategy based on initialization models for the topology and the operating conditions is described to enhance the robustness of the optimization method. Application of the proposed model yields flowsheet designs with improved savings in capital and utilities cost.
\end{abstract}

Keywords: Synthesis, crystallization, paraxylene separation process, optimization

\section{Introduction}

The C8 aromatic isomers are very difficult to separate by conventional distillation processes because of their close boiling points, which in the particular case of paraxylene and metaxylene differ by less than $1^{\circ} \mathrm{C}$. However, paraxylene can be readily separated from other xylene components taking advantage of its higher freezing point. In this way, separation processes based on crystallization and liquid/solid separation equipment are an alternative technology, allowing paraxylene to be solidified and recovered in crystal form at a temperature at which the other isomers are present in liquid phase. This process generally consists of multiple processing stages in series and parallel for creating and separating solids, where crystallizers, liquid/solid separation devices such as different types of centrifuges, drums, heat exchangers and refrigeration systems are the most important equipment that is used. Although separation processes based on crystallization technology have become increasingly important in chemical industry, they have received much less attention in the optimization area, where most efforts have been focused on distillation systems (Barttfeld M. et al., 2002). We address the synthesis and optimization of separation processes based on crystallization where

*Author to who correspondence should be addressed: grossmann@cmu.edu 
major decisions involve selecting: (a) number of crystallization stages, (b) type, number and size of equipment at each stage and interconnections between unit operations, (c) temperature levels for crystallizers, heat exchangers, refrigeration system and flows in the system, and (d) alternatives for the return of recycles.

\section{Crystallization superstructure}

Figure 1 illustrates a general topology for a n-stage crystallization process. The feed stream contains the following 4 components in the liquid phase: paraxylene, metaxylene, orthoxylene and ethylbenzene. However, since paraxylene is crystallized during the process, we treat paraxylene in the liquid and solid phase as two different components that can be present in each process stream. In order to guarantee that only paraxylene be solidified, the eutectic point (temperature at which a second solid phase will form) is used as a lower bound on the process temperature. In addition, the paraxylene incipient crystallization temperate (ICT) is used to satisfy certain operating restrictions. Since crystallizers represent the most important equipment and involve significant capital and operating cost, a superstructure is embedded into the model to consider all the alternatives for series/parallel arrangements for a given number of crystallizers. Figure 2 depicts the superstructure for a set of crystallizers.

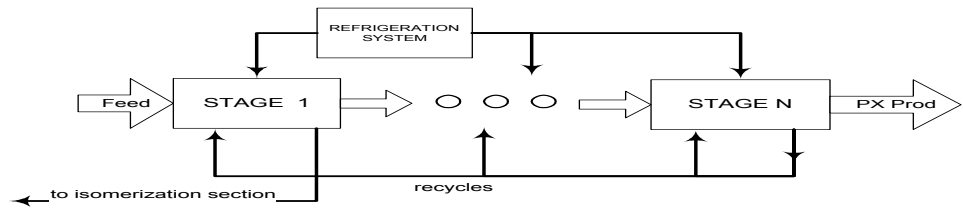

Figure 1. A general n-stage separation process

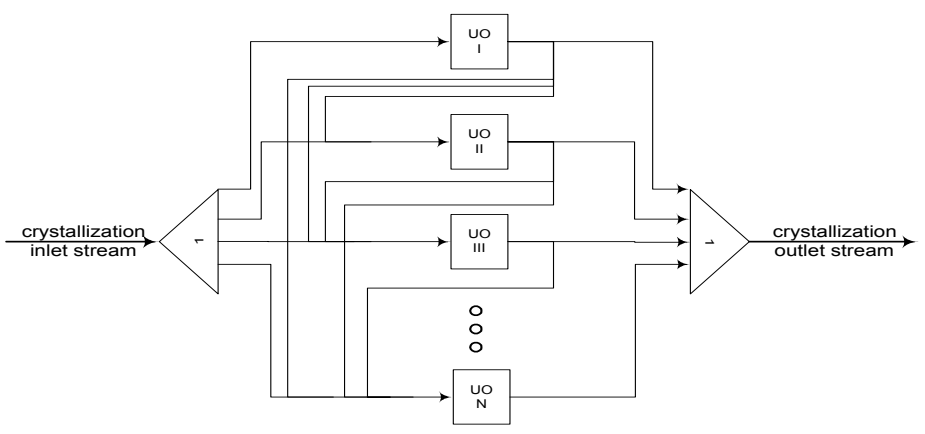

Figure 2. A general superstructure for a crystallization process

\section{MINLP formulation}

This section describes the proposed MINLP model for the synthesis problem. Since this process involves multicomponent streams, we define individual component flows $f_{s, c}$ for each stream. As a result, bilinear terms are only required for the material balances in the splitters. 
Solubility. The non-linear solubility correlation $f_{s o l}$ predicts the maximum liquid concentration of paraxylene in each stream $s$ at a temperature $T_{s}$. Consequently, the amount of paraxylene in the liquid and solid phases is explicitly defined for most of the process streams, which is a central aspect of the problem. However, we have to consider two possible alternatives: (i) the solubility predicts the exact amount of paraxylene in the liquid phase or (ii) the solubility predicts a concentration of liquid paraxylene that cannot be met with the current stream composition. To deal with the second case, we define constraints (2) and (3) and the variable $f c_{s}$ which becomes positive and allows equation (4) to be always satisfied as equality. Equation (3) represents a smooth approximation of the " $\max [0, \mathrm{x}]$ " function (Biegler et al. 1987) which defines the exact amount of paraxylene that would be required to meet the solubility prediction.

$$
\begin{aligned}
& s o l_{s}=f \operatorname{sol}\left(T_{s}\right) \quad \forall s \in S \\
& s c_{s}=\operatorname{sol} \sum_{c \in C} f_{s, c}-f_{s, c^{L}}-f_{s, c^{s}} \quad \forall s \in S \\
& f c_{s}=\frac{\sqrt{s c^{2}}}{2}+\frac{s c_{s}}{2} \quad \forall s \in S \\
& f_{s, c^{L}}=\operatorname{sol}_{s}\left(\sum_{c \in C^{L}} f_{s, c}+f_{s, c^{L}}\right)-f_{c_{s}} \quad \forall s \in S
\end{aligned}
$$

Material balances. Equation (5) defines the mass balance for those components that can only be in liquid phase whereas equation (6) forces the mass balance of liquid and solid material for the component to be separated.

$$
\begin{aligned}
& \sum_{s \in S_{u}^{I}} f_{s, c}=\sum_{s \in S_{u}^{O}} f_{s, c} \quad \forall u \in U, c \in C^{L} \\
& \sum_{s \in S_{u}^{I}} f_{s, c^{L}}+f_{s, c^{s}}=\sum_{s \in S_{u}^{O}} f_{s, c^{L}}+f_{s, c^{s}} \quad \forall u \in U
\end{aligned}
$$

Additionally, the condition that the streams leaving a splitter have the same ratios in flow for each component is enforced by constraint (7).

$$
f_{s, c}=f_{s^{\prime}, c} e_{u, s} \quad \forall u \in U^{S P}, c \in C, s^{\prime} \in S_{u}^{I}, s \in S_{u}^{O}
$$

Stream density. A linear function $f d$ of the stream temperature is used to predict the density of each stream, which is then utilized to size the drums used in the separation process.

$$
\text { dens }_{s}=f d\left(T_{s}\right) \quad \forall s \in S
$$

Heat balances. Specific non-linear functions $f e$ are used to compute the heat transfer taking place in each process unit.

$$
f e\left(f_{s_{u}, c}, T_{s_{u}}, Q_{u}\right)=0 \quad \forall u \in U
$$

Equipment sizing. The area or the volume of the equipment $\left(\right.$ size $\left._{u}\right)$ is determined through a nonlinear function $f v$. Minimum and maximum capacities are enforced for those units belonging to the process, i.e. $Y_{u}=1$.

$$
\begin{aligned}
& \text { size }_{u}=f v\left(f_{s_{u}, c}, T_{s_{u}}, \text { dens }_{s_{u}}\right) \quad \forall u \in U \\
& V_{u}^{\min } Y_{u} \leq \operatorname{size}_{u} \leq V_{u}^{\max } Y_{u} \quad \forall u \in U
\end{aligned}
$$


Liquid/solid separation devices. The performance of the liquid/solid separation devices is determined through Constraint (12). In this way, the amount of component leaving the unit in each outlet stream will depend on certain equipment parameters and on the stream feeding the unit, which is considered in the non-linear function $f s$.

$f_{s^{\prime}, c}=f_{s}\left(f_{s, c}\right) \quad \forall u \in U^{S L}, s \in S_{u}^{I}, s^{\prime} \in S_{u}^{O}$

Unit operating limits. Certain operating conditions over minimum and maximum liquid and solid flowrates that some units are able to handle are modeled in constraint (13). In addition, this constraint forces all the streams related to particular unit to be zero when the piece of equipment is not included in the process.

$$
F_{u, c}^{\min } Y_{u} \leq f_{s, c} \leq F_{u, c}^{\max } Y_{u} \quad \forall u \in U, s \in S_{u}^{I}
$$

Production target. The total amount of product and the minimum component concentration to be recovered is imposed through equations (14) - (15).

$$
\begin{aligned}
& \sum_{c \in C} f_{s^{p r o d}, c} \geq P T \\
& f_{s^{p r o d}, c^{L}}+f_{s^{\text {rord }}, c^{s}} \leq p c \sum_{c \in C} f_{s^{\text {rrod }}, c}
\end{aligned}
$$

Objective function. The nonlinear function $f_{c}$ computes the annualized profit considering product revenues, feed cost, recycled material price (to the isomerization section), utilities cost (steam and electricity) and capital investment. In order to reflect economies of scale, we use concave cost functions for capital costs (Douglas 1988; Biegler et al., 1997).

$\operatorname{Min} \quad Z=f c\left(f_{s^{\text {feed }}, c}, f_{s^{\text {prod }}, c}, f_{s^{\text {regede }}, c}, Q_{u}\right.$, size $\left._{u}\right)$

\section{Solution strategies}

Taking into account the complexity of the separation process, the large number of equipment and flows and the highly nonlinear equations involved, the general mathematical formulation introduced in the previous section generates a large and complex MINLP model, where even feasible solutions are difficult to find. In order to enhance the model robustness without compromising the optimality of the solution, we use different strategies that significantly improve the model behaviour and, at the same time, generate better initial solutions for the nonlinear optimization method. The most important solution strategies are described below.

1. Sequential procedure for solving the entire optimization problem. The main idea here is to gradually incorporate aspects of increasing complexity through the following sequential optimization steps. (i) Solve the NLP model where all discrete variables are fixed to 1 and linear solubility correlations described below are used, (ii) Correct solubility predictions by using exact non-linear correlations (NLP model), (iii) Solve the MINLP model by only fixing to 1 the discrete variables related to liquid/solid separation devices (iv) Solve the MINLP model considering all the discrete decisions. In this way, different decisions are sequentially made and a significant improvement in the convergence is obtained. It should be noted that 
although we are considering the entire optimization problem in the last step, a very good initial solution is provided by performing the previous steps.

2. Linearization of solubility correlations. The solubility is a central aspect of the problem that involves highly non-linear and non-convex correlations. Linear approximations are used in the first step of the sequential procedure in order to find a good initial solution which is then corrected in following steps by using the original nonlinear solubility correlations.

3. Super-equipment definition. Since equipment working in parallel have fixed capital cost and capacity, they can be treated in the model as a single aggregated equipment with the discrete variables defining the number of individual units. In this way, the number of process streams and, consequently the model size is greatly reduced.

4. Minimum equipment requirement. The proposed superstructure comprises a large number of process units and 0-1 variables defining whether they are used or not in the optimal solution. Taking advantage of the process knowledge and the unit operating limits, it is possible to estimate the minimum number of a particular type of equipment that would be required in any feasible solution. In this way, some discrete variables can be fixed to 1 a priori, which greatly reduces the CPU time.

\section{Numerical results}

The proposed MINLP formulation together with the solution strategies described above were successfully used to conduct studies for the design and synthesis of different schemes for the crystallization process. Figure 3 shows the generic flowsheet for a crystallization process design. The MINLP formulation of our actual problem involves 20 binary variables, 1381 continuous variables and 1229 constraints and was solved on a Pentium IV PC using CONOPT for the NLP solver and CPLEX 8.1 for the MILP solver in GAMS 21.2/DICOPT++ (Viswanatahn and Grossmann, 1990). The total solution time for the sequential procedure was 33 seconds using the heuristic termination that stops the search when the objective function of the NLP subproblems starts to deteriorate. Both variable and annualized capital costs contributed significantly $(>10 \%)$ to the objective function. The entire process comprises multiple crystallizers in series and parallel and many liquid/solid separation devices with different configurations, most of them working at full capacity.

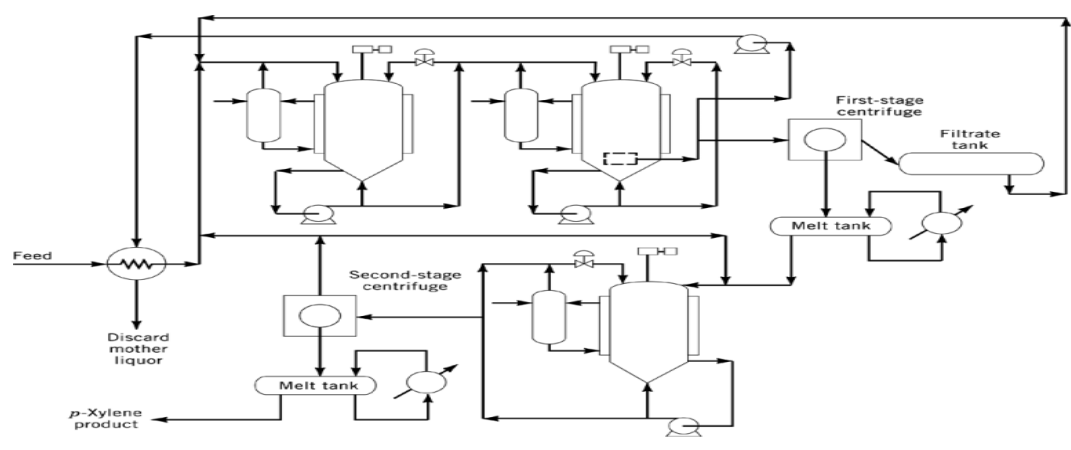

Figure 3. General flowsheet for a multiple-stage crystallization design 


\section{Conclusions}

An efficient MINLP approach for the optimal design and synthesis of paraxylene separation processes based on crystallization technology has been presented. Different designs and operations conditions were evaluated and significant savings in capital and utilities were obtained with low computational requirements.

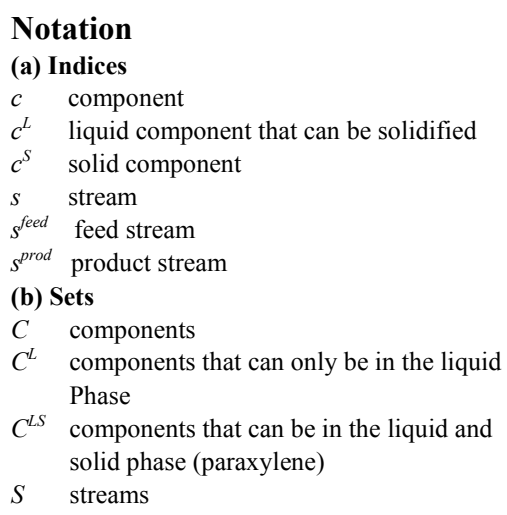

(c) Parameters

$F^{\min }{ }_{u, c}$ minimum flowrate of component $c$ in unit $u$

$F_{u, c}^{\max }$ maximum flowrate of component $c$ in unit $u$

$V^{\min }{ }_{u}$ minimum size or capacity for unit $u$

$V^{\max }{ }_{u}$ maximum size or capacity for unit $u$

(d) Variables

dens $s_{s}$ density of stream $s$

$e_{u, s} \quad$ split fraction corresponding to outlet stream $s$ of splitter $u$

$f_{s, c} \quad$ flowrate of component $c$ in stream $s$

$f c_{s}$ exact amount of component required to meet solubility prediction

$Q_{u} \quad$ heat transfer in unit $u$

size $_{u}$ total area or volume corresponding to unit $u$

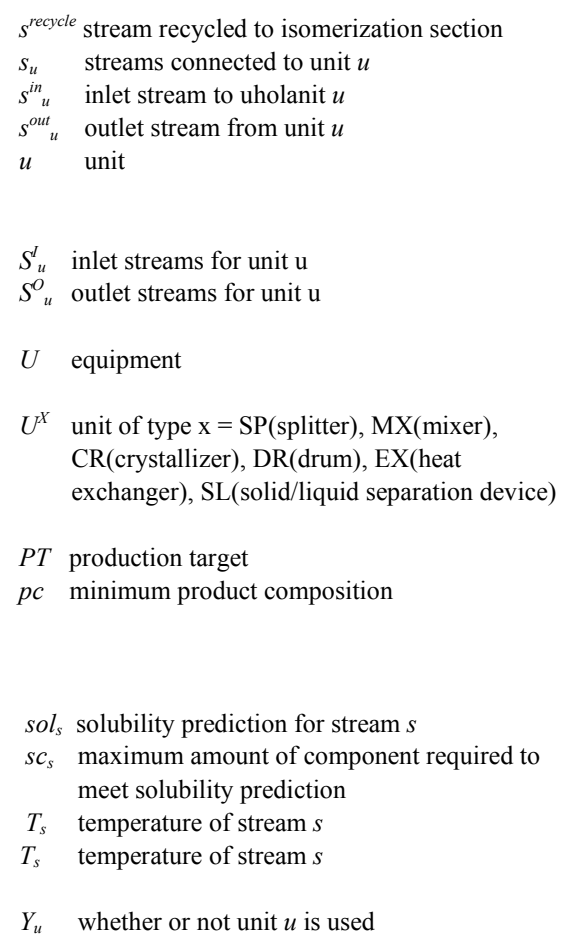

\section{Acknowledgments}

The authors would like to thank BP for financial support of this project

\section{References}

Barttfeld M., Aguirre P.A. and Grossmann I.E., 2004, A decomposition method for synthesizing complex column configurations using tray-by-tray GDP models. Computers and Chemical Engineering, 28, 11, 2165.

Brooke A., Kendrick D., Meeraus A. and Raman R., 1997, GAMS language guide, Release 2.25, Version 92. GAMS Development Corporation.

Biegler L.T., Grossmann I.E. and Westerberg A.W., 1997, Systematic methods of chemical process design. Prentice Hall, New Jersey.

Douglas J.M., 1988, Conceptual design of chemical processes. McGraw-Hill, New York.

Viswanathan J. and Grossmann I.E., 1990, A Combined penalty function and outer approximation method for MINLP optimization, Computers and Chemical Engineering 14, 769. 